JOURNAL OF LAW, TECHNOLOGY \& THE INTERNET • VOLUME $11 \cdot$ NO. $5 \cdot 2019-2020$

\title{
CONSTITUTIONS AND BLOCKCHAINS: COMPETITIVE GOVERNANCE OF FUndAMENTAL RULE SETS
}

\author{
Eric Alston ${ }^{* \dagger}$
}

In the context of private ordering - where rule sets are relatively fluid, centrally controlled, and exist in the shadow of law and regulation-developing generalizable insights about comparatively superior governance mechanisms is difficult. I shed light on this question by characterizing cryptocurrency blockchains as a type of constitutional rule set that both defines and legitimizes the activities supported by the underlying distributed ledger technology. More specifically, I argue that cryptocurrency blockchains have led to new forms of competition in private governance, which include exit costs and citizenship rules as important competitive margins. My analysis not only identifies the choices in constitutional governance to which proposed cryptocurrency blockchain changes are analogous, but it also highlights the competitive gains expected to result from these changes. I further consider both the trade-offs in governance created by competition between cryptocurrency blockchains and the surprising ways in which these unique competitive margins may influence downstream outcomes.

\footnotetext{
${ }^{*}$ Scholar in Residence, Finance Division, Faculty Director, Hernando de Soto Capital Markets Program Leeds School of Business, University of Colorado Boulder

${ }^{\dagger}$ For helpful comments and suggestions, I thank participants at the following seminars and conferences: King's College London's Center for the Study of Governance and Society's Symposium on Self-Governance, Canadian Law and Economics Association Annual Conference, Institute for Humane Studies and Center for Growth and Opportunity's Frontiers in Permissionless Innovation Seminar, and North Dakota State University's Conference on Research and Practice on Blockchain. For research support, I gratefully acknowledge funding from the Center for Growth and Opportunity at Utah State University, the Hernando de Soto Capital Markets Program, and the Leeds Business School at CU Boulder. I also thank Lindsay Vanlerberg and Derek Fenton for valuable research assistance. All errors are my own.
} 


\section{CONTENTS}

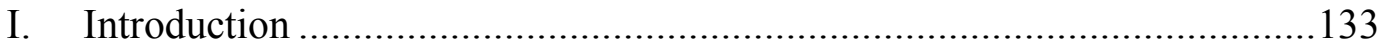

II. Constitutional Design: Rule Entrenchment, Citizenship, and Amendment.135

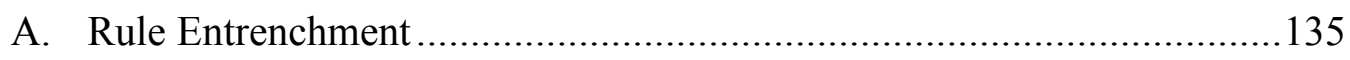

B. Citizenship Rules (Exit \& Entry Costs)............................................139

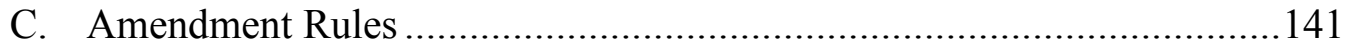

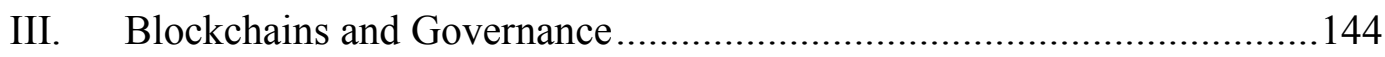

A. Blockchains and Constitutions ........................................................... 149

B. Cryptocurrency Governance Debates................................................154

1. Ethereum and Ethereum Classic .............................................. 155

2. Bitcoin v. Bitcoin Cash ................................................................. 156

3. Lightning Network (BTC) \& Plasma/Sharding (ETH) .....................157

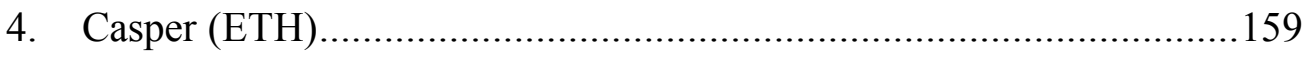

5. Emergent Governance Competitors: Tezos \& EOS...........................161

C. Competitive Constitutional Governance on Blockchains.......................163

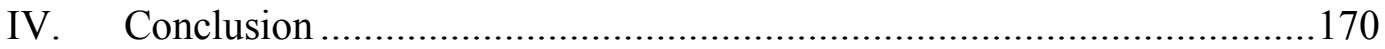




\section{INTRODUCTION}

Constitutions are the most famous example of what legal scholars refer to as secondary rules. More colloquially, secondary rules are the rules about making rules. $^{1}$

In the case of constitutions, these rule sets determine how laws are enacted, administered, and adjudicated in a given society. Constitutional rules are thus accorded a high level of importance in a given nation's legal system because they are quite literally the foundation upon which all subsequent legislation and government action rest. Because of constitutional rules' fundamental nature, these rules are by design less subject to change than ordinary legislation. In other words, constitutional rules are typically the most rigid rule set in a given legal system. Nonetheless, constitutions vary as to the means and ease by which they can be amended. Thus, the comparative flexibility of a given constitution is an institutional design choice that has long been treated in the scholarship on comparative constitutional design, which has linked flexibility both to endurance and to a diminished need for informal measures of constitutional adjustment.

Where there is a distinct understanding of the fundamental rules governing human interactions - perhaps because of the lack of a uniform standard of comparison and public availability of underlying policies - is in the context of private governance. This may be in part because one of the benefits of a firm structure is that decisions regarding policy change are typically validated through a centralized process, as opposed to a democratic one, which inherently creates less need for "governance of governance" in that the "law" is whatever the central firm authority says it is. From an economic perspective, the firm provides the quintessential example of private governance. Previous scholars such as Coase and Williamson have demonstrated how governance within the firm is an essential field of study for understanding economic outcomes. ${ }^{2}$ The ubiquity of the firm as an organizational structure governing economic production has a corollary implication: the choice of private institutions by various firms provides a margin for competition. However, the extent to which firms can freely compete in terms of internal governance and the agreements struck with different employees and suppliers is an open question in a world of increased legislation and regulation of contractual agreements, especially in the case of publicly-traded companies.

\footnotetext{
${ }^{1}$ See generally Lionel Adolphus Hart \& LeSlie Green, The Concept of LaW (2012).

${ }^{2}$ See Ronald Coase, The Nature of the Firm, 4 ECONOMICA 386-405 (1937); Oliver E. Williamson, The Theory of the Firm as Governance Structure: From Choice to Contract, 16 JouRnaL OF ECONOMIC PERSPECTIVES 172-95 (2002).
} 
In a world of regulated and centralized firms, how do scholars derive insights about the comparative benefits of different choices in private governance? The emergence of blockchains as the technology underlying cryptocurrencies provides a fruitful context in which to examine this question. The example of the different tensions faced by Ether and Bitcoin, and the different responses in terms of changes to the fundamental underlying structure of the blockchains supporting the two cryptocurrencies, suggests value in comparing how different mechanisms of blockchain governance result in different outcomes for cryptocurrencies and their intended functions. Using insights from constitutional design generally, and amendment processes specifically, I explore the differences in blockchain governance mechanisms in this article. I conclude that blockchains can be understood as a type of constitutional rule set that both defines and legitimizes the activities supported by permissionless distributed ledger technology. ${ }^{3}$ In particular, the exit costs and amendment processes defined by the "constitutional" regimes that blockchains define create a unique competitive margin that will shape the comparative success of a given cryptocurrency.

I proceed by first reviewing the existing scholarship on constitutional processes and by identifying how ubiquitous constitutional design features (amendment rules, rule entrenchment, and citizenship provisions) can each play a role in facilitating the objectives of those engaged in a rule-based collective decision-making process like those governed by permissionless blockchains. I then provide a brief description of the blockchains supporting major cryptocurrencies, identifying the ways in which the underlying network rules are similar to and distinct from constitutional rules. Next, I compare existing and proposed governance structures for blockchains, especially those supporting the cryptocurrencies Bitcoin and Ether, but also other permissionless use cases. My analysis identifies not only the choices in constitutional governance to which proposed blockchain changes are analogous but also the competitive benefits and costs that result, with a view to the intended functions of a given blockchain. Finally, I consider the trade-offs in governance that competition between cryptocurrency blockchains creates, examining how these unique competitive margins can influence downstream outcomes, and predicting that membership rules, rule entrenchment, and amendment processes will become increasingly salient governance choices.

\footnotetext{
${ }^{3}$ Other scholars have contemporaneously drawn related comparisons to mine. See, e.g., Shruti Rajagopalan, Blockchain \& Buchanan: Code As Constitution (2019) (in James Buchanan, Theorist of Political Economy and Social Philosophy, (Palgrave MacMillan 2019); see also Alastair Berg, Chis Novak, \& Mikayla Novak, Blockchains and Constitutional Catallaxy (Dec. 4, 2018), available at: https://ssrn.com/abstract=3295477 or http://dx.doi.org/10.2139/ssrn.3295477.
} 


\section{Constitutional Design: Rule Entrenchment, Citizenship, AND AMENDMENT}

The principal bulk of our behavior is not governed directly by constitutional rules. Instead, our behavior is governed by the legislative, executive, and judicial output of public organizations defined by these constitutional rules. It is in this sense that "the rules about making rules" are secondary, as Hart described them. ${ }^{4}$ Our primary day-to-day activities and the majority of our disputes and prohibited actions are governed by primary rules; secondary rules instead define the system that writes, administers, and adjudicates the primary rules governing our behavior. This is because there are significant benefits to agreeing to the means by which subsequent rules will be defined and changed. If, instead, every rule change also required a debate over the means by which the rule would be defined, creating new rules would be much harder, if not impossible. ${ }^{5}$ Put differently, the game cannot be played if no one agrees on the underlying rules. This is the essential argument for the ubiquitous emergence of constitutional rule sets in governance systems around the world. ${ }^{6}$ Within these rule sets, though, there are also more specific constitutional design features that reduce the costs of collective decision making at scale: rule entrenchment, citizenship (or membership) rules, and amendment processes. Both the multi-tiered nature of constitutional rules more generally, as well as these design features more specifically, share intended functions with blockchains that reveal important governance tradeoffs for different cryptocurrency communities.

\section{A. Rule Entrenchment}

The first ubiquitous constitutional design feature is that of rule entrenchment, or insulation. The reasons for the entrenchment of rules follow directly from the logic of secondary rules more generally. Leaving decision rules up for grabs can greatly stymy group decision making due to the increased costs and strategic incentives already discussed. Even if a rule is not given heightened protection from change as compared to the ordinary decision rules in a given group, decision-making procedures tend to limit a given meeting to a known set of topics,

\footnotetext{
${ }^{4}$ See Hart \& Green, supra note 1.

${ }^{5}$ A multi-tiered rule process focuses the margins of political competition on a more narrow set of issues, once the rules of the game have been agreed upon. See, e.g., James M. Buchanan, The Relatively Absolute Absolutes, in THE LOGiCAL Foundations OF CONSTITUTIONAL LiBERTY 44850 (Liberty Fund, 1999); see also Jonathan Riley, Constitutional Democracy as a Two-Stage Game, in Constitutional Culture and Democratic Rule 147-50 (Cambridge University Press, 2001).

${ }^{6}$ See James M. Buchanan \& Gordon Tullock, The Calculus of Consent 60-80 (University of Michigan Press, 1962) (discussing why constitutional rule sets could emerge as the result of purely self-interested decisions among constituents).
} 
in part to prevent the decision rules from being subject to opportunistic change. ${ }^{7}$ However, this specific procedural rule, limiting group decisions to the matters defined ex-ante, raises a more general design question surrounding secondary rules: which rules have to be defined before ordinary decision-making can proceed? This begets the notion that some rules are properly constitutional. The rules about making rules have to be defined before decisions about ordinary rules can be made. However, secondary rules also necessarily empower and constrain the individuals who make decisions on behalf of the group to ensure that decision-making follows a known and defined process.

The fact that the secondary rules both define and constrain the individuals uniquely empowered as compared to ordinary group members has led to the additional institutional design choice that is nearly ubiquitous in these contexts: insulation of secondary rules from the ordinary processes of collective decisionmaking. ${ }^{8}$ In practice, this typically takes two forms. First, changes to secondary rules typically require a higher degree of consensus among group decision-makers as compared to changes to ordinary rules. The canonical example of this in practice is the requirement in political systems that constitutional amendments require a legislative supermajority to occur. ${ }^{9}$ The second form of rule entrenchment, is the involvement of a larger portion of the group, or a distinct subset of group members, in such decisions. ${ }^{10}$ Clear examples of rule entrenchment from constitutional design worldwide are the involvement of subnational units in constitutional amendments

\footnotetext{
${ }^{7}$ See John W. Patty \& Elizabeth Maggie Penn, The Legislative Calendar, 48 Mathematical AND COMPUTER MODELLING 1590-91,1599-1600 (2008) (discussing the necessity of a general understanding of legislative schedule setting, given its ubiquity in practice).

${ }^{8}$ Options in practice of more less stringent amendment rules abound. If occurring via legislative change, adjustment of the threshold required for passage is a common choice, with a higher threshold accordingly making passage more difficult, and vice versa. Another margin defining the difficulty of amendment involves the extent to which different stakeholders' input is required in order for amendment to occur; if an amendment can only occur after it has successfully passed the legislative threshold and a popular referendum, this is more difficult than requiring either one of those options. See Bjørn Erik Rasch \& Roger D. Congleton, Amendment Procedures and Constitutional Stability, 12 Dem. Const. Design \& Pub. Pol'y: Analysis And Evidence 536-49 (2006) (discussing options in practice of less stringent amendment rules abound. If occurring via legislative change, adjustment of the threshold required for passage is a common choice, with a higher threshold accordingly making passage more difficult, and vice versa. Another margin defining the difficulty of amendment involves the extent to which different stakeholders' input is required in order for amendment to occur; if an amendment can only occur after it has successfully passed the legislative threshold and a popular referendum, this is more difficult than requiring either one of those options).

${ }^{9}$ See Tom Ginsburg \& James Melton, Does the Constitutional Amendment Rule Matter at All? Amendment Cultures and the Challenges of Measuring Amendment Difficulty, 13 INT'L J. CONST. L. 691 (2015).

${ }^{10}$ See id. at 692.
} 
(like the role of states governments in the United States) ${ }^{11}$ or the requirement that proposed constitutional amendments pass a public referendum before enactment. ${ }^{12}$ The formal articulation of core group principles and beliefs also frequently receive this level of entrenchment, ${ }^{13}$ even if the entrenchment of these principles is not strictly necessary from the functional perspective of ordinary group decisionmaking.

Instead of the inadequacy of a given constitution eventually leading to it being overturned and replaced, a lower cost outcome is the adjustment of the constitution to prevent outright constitutional rupture. ${ }^{14}$ This makes the exact level of rule entrenchment an important constitutional design question. ${ }^{15}$ Because of the relative entrenchment of constitutional rules compared to ordinary legislation, changing them in practice tends to provide an expressive function in signaling that a cornerstone aspect of governance is subject to potential change. ${ }^{16}$ Meaning, the very act of amending a constitution raises the salience of the particular areas in question (as well as inflaming "constitutional passions," independent of the underlying political or social questions at issue). ${ }^{17}$ The ability to amend a fundamental charter also provides for dynamic legitimization; if those currently governed by a given constitution can change it, then they are more likely to view the constitution as reflecting their needs and goals surrounding governance. This concept of the ongoing "fit" between the written constitutional document and societal preferences surrounding governance is one that comes up against the strength of governance preferences today, versus the need for the intertemporal ability to adjust this fit. Which aspects, if any, of the constitution should be insulated to the point that they are never subject to future adjustment?

\footnotetext{
${ }^{11}$ See Rasch \& Congleton, supra note 8, at 326.

12 See Ginsburg \& Melton, supra note 9, at 692.

${ }^{13}$ See Heinz Klug, Model and Anti-Model: The United States Constitution and the Rise of World Constitutionalism, 2000 WIS. L. REV. 597, 601 (2000).

${ }^{14}$ See generally Richard Albert, The Expressive Function of Constitutional Amendment Rules, 59 MCGILL L.J. 225 (2013) (discussing the corrective nature of constitutional amendment procedures and safeguards those procedures provide); see also SANFORD LEVINSON, RESPONDING TO IMPERFECTION: THE THEORY AND PRACTICE OF CONSTITUTIONAL AMENDMENT (Sanford Levinson ed., 1995).

15 Despite many similar and identical amendment processes around the world, there is significant variation in the extent of change that a given level of constitutional flexibility yields in practice. This suggests that rule entrenchment, like many constitutional design questions, interacts in complex ways with the political and social milieu within which it is enacted and implemented. See Ginsburg \& Melton, supra note 9, at 692-701.

${ }^{16}$ See Rasch \& Congleton, supra note 8, at 325-26.

${ }^{17}$ Rosalind Dixon \& Tom Ginsburg, Deciding Not to Decide: Deferral in Constitutional Design, 9 INT'L J. CON. L. 636, 636-72 (2011) (examining how different constitutional amendments and methods of amending are impacted by various social and political considerations).
} 
The distinction between adherence to the letter of constitutional law, and adherence to the ongoing societal governance beliefs that animated the creation of the constitutional order writ large, has been well studied by constitutional scholars. Put simply, constituents in general, and political actors in particular, are guided by their beliefs as to what the constitution should (and should not) achieve. ${ }^{18}$ Just as sufficiently reprehensible government action itself can trigger regime change, sufficient change to the constitutional definition of governments (and its checks and balances) has long been argued as capable of annihilating or eliminating the existing constitutional order altogether. ${ }^{19}$ The justification for making some provisions unamendable clearly spells out the distinction between the unwritten constitutional principles that are crystallized through a constituent process into the written constitution: "the immutability of the principles . . marked out a normative core that defined the constitutional identity of the polity." ${ }^{20}$ Unamendability, as the most extreme form of rule entrenchment, thus serves a profound expressive function, albeit one that is fundamentally anti-democratic intertemporally in the case of public constitutional orders.

Although unamendability provides a clear link between the formal constitution itself and the constitutional principles animating the creation of the written document, the existence of an underlying set of constitutional beliefs is not limited to contexts where a subset of fundamental rules are unamendable per se. Fundamental beliefs about blockchains' immutability and the need to punish bad actors stand as examples of these constitutional beliefs in the context of cryptocurrency communities, as the discussion in section $\mathrm{V}$ emphasizes.

Constitutional rulesets' supremacy has an important corollary: exiting a national constitution's ruleset, or in other words, revoking one's own citizenship,

\footnotetext{
18 See Norman Schofield, Architects of Political Change: Constitutional Quandaries AND SOCIAL CHOICE THEORY 260-62 (Stephen Ansolabehere ed., 2006); see generally Eric Alston, Lee Alston, \& Bernardo Mueller, Institutional AND ORGANIZATIONAL ANALYsis: CONCEPTS \& APPLICATIONS 273-313 (2018).

${ }^{19}$ Carl Schmitt's 1928 critique of the paradox created by substantively unconstrained amendment procedures has been linked to the emergence of unamendable provisions in the German Basic Law of 1949. See CARL SchmitT, Constitutional TheORy 150-51 (Jeffery Seitzer ed. \& trans., Duke University Press 2008); e.g., Ulrich K. Preuss, The Implications of Eternity Clauses: The German Experience, 44 ISR. L. REV. 439, 439 (2011). (explaining that the emergence of the Nazi regime that was so at odds with the core constitutional principles that gave birth to the Weimar Republic, led drafters of the Basic Law after World War II to adopt specific provisions that the fundamental aspects of the structure of the government could not be altered through the government's power, effectively getting rid of a constitutional basis for amending these elements and requiring the wholesale replacement of Basic Law by Germans through extra-constitutional means).

${ }^{20}$ Preuss, supra note 19, at 441.
} 
often imposes significant costs. Moving from state to state can allow a citizen considerable choice as to the rules governing their behavior; the rules of the United States Constitution, however, do not change from state to state. The higher the exit costs, the more important the process of dynamic constitutional legitimization discussed thus far becomes, and vice versa, because, without a viable ability to change the rulesets that govern one's life, the only alternative is to exit the ruleset. ${ }^{21}$ Amendment rules at least partially address this problem and create a space within which the constitution can be changed. Constitutional amendment procedures can range from the extreme of making the constitution unamendable to making constitutional change subject to the same requirements as ordinary legislation. At the extreme of unamendability, constituents would have considerable certainty as to the finality of constitutional rules governing them at any given time, while at the other, constituents could easily adapt the constitution to suit new circumstances and changing beliefs surrounding governance. ${ }^{22}$ In practice, modern national constitutions strike a balance between these two extremes. Even if the drafters intend to lean towards unamendability, one scholar notes that the need for constitutional amendment is so fundamental that "all constitutions admit the possibility of amendment," 23 in great part because the option of exit is so costly for most individuals governed by a given constitution. For most citizens of nationstates around the world, they are a member of a given constitutional order not by choice, but by the consequence of birth.

\section{B. Citizenship Rules (Exit \& Entry Costs)}

Another key design feature in order for a secondary rule set to function in governing a given group is that of membership: what characteristics define who is a member of the group, and how can members leave the group (whether voluntarily or not)? In practice, these requirements can take the form of costs imposed upon joining or leaving the group: entry and exit costs. Entry costs are probably the most familiar to political theorists in the form of citizenship requirements: what conditions must be satisfied in order for a given individual to be able to participate

\footnotetext{
${ }^{21}$ Exit costs have long been identified as a central determinant of the institutions of governance that emerge in both public and private organizations. See Albert HirSchman, Exit Voice AND LOYALTY: RESPONSES TO DECLINE IN FIRMS, ORGANiZATIONS, AND STATES 4-7 (1970). These costs have also been identified as important determinants of governance outcomes in the private sector; see, e.g., Colin Mayer, Corporate Governance and Performance, 21(1) J. L \& Soc'Y. 152, 156 (1997); John Coffee Jr., Accountability and Competition in Securities Class Actions: Why Exit Works Better Than Voice, 30 CARDOZO L. REV. 407, 409-10 (2008).

22 Sanford V. Levinson, Designing an Amendment Process, in Constitutional Culture and Democratic Rule 271, 272-274 (2001).

${ }^{23}$ Andrew Roberts, The Politics of Constitutional Amendment in Postcommunist Europe, 20 Const. POL. ECON. 99, 99 (2009) (emphasis mine).
} 
in a political system with the standard rights and duties accorded to all other citizens? ${ }^{24}$ Entry requirements can be a screen for well-justified as well as dubious reasons: conditioning membership in a homeowners' association on actual homeownership verges on the tautological, whereas conditioning such membership on race or ethnicity would rightly be broadly condemned in society today. Entry costs can, therefore, be conditioned on reasons related to the good of the organization itself, or for reasons unrelated to the strength or sustainability of the organization.

Conditioning membership on a certain level of commitment or characteristics can shape membership or members' incentives in ways that improve governance outcomes. If the costs are high enough to become a member, other members can receive more assurance as to the level of desire for membership on the part of new members. If one has made a costly investment in membership, one's incentives to influence governance outcomes for the better are likely stronger than in the case of a group that it costs nothing to join.

Just as becoming a member of the organization can be more or less costly to a given individual, so too can departing from the organization. For instance, taxes imposed on Americans revoking their U.S. citizenship are, in part, an attempt to condition the departure costs of high wealth citizens who may be incentivized to relinquish citizenship in search of a cheaper tax burden elsewhere. ${ }^{25}$ The extended process associated with changing citizenship also temporally delimits the ability of individuals to exit - one can effectively only change citizenship after a considerable amount of time has passed. The functional effect of such financial and temporal exit costs is that, by making exit more costly, an organization's members are more likely to engage in the costly process of governance. If a given "citizen" can't leave, they might as well influence the rules that will govern them. Currently, cryptocurrency community members face relatively low entry and exit costs, something that will likely change as cryptocurrency blockchains continue to compete on governance choices directly.

\footnotetext{
${ }^{24}$ See Kim Rubenstein \& Niamh Lenagh-Maguire, Citizenship and the Boundaries of the Constitution, in Comparative Constitutional LaW 143, 143 (2011); see also Patrick Weil, Access to Citizenship: A Comparison of Twenty-Five Nationality Laws, in CITIZENSHIP TODAY: GLOBAL PERSPECTIVES AND PRACTICES 17, 17 (2001).

25 See William Thomas Worster, The Constitutionality of the Taxation Consequences for Renouncing U.S. Citizenship, 9 Fla. TAX REV. 921, 923 (2010).
} 


\section{Amendment Rules}

Ultimately, though, these specific constitutional design questions interact with one another in deep structural ways. The membership trade-offs influenced by entry and exit costs are also shaped by the choice of amendment rules: a constitution can be comparatively more flexible or rigid, depending upon how difficult it is to amend, which is itself a question of the extent of rule entrenchment originally chosen by constitutional drafters. ${ }^{26}$ Amendment rules have been characterized as necessarily corrective, recognizing the likelihood that a constitution will prove deficient in the light of unforeseen future events. Ultimately, though, the need for constitutional change is a function of how well initial constitutional design choices dynamically facilitate economies of scale in governance while simultaneously minimizing agency costs.

Beyond the most basic structural trade-off between flexibility and rigidity, constitutional scholars have deepened the comparative understanding of choices of amendment rules. Thus, the trade-off between flexibility and rigidity has also been characterized as trading off between stability and flexibility; ${ }^{27}$ but this characterization of stability is more focused on the stability of the underlying rule set, ${ }^{28}$ as opposed to the stability of governance overall. The underlying intuition is a simple one: a constitution that faces more barriers to adjustment is less likely to be adaptable to changing circumstances in society, and hence, is more likely to provoke the need for a wholesale constitutional overhaul in cases where it does not fit the needs and beliefs surrounding governance in society. Nonetheless, too much flexibility at some level undermines the basic principle of constitutionalism as a set of stable limits on ordinary politics. ${ }^{29}$ Because of the complexity of these trade-

\footnotetext{
${ }^{26}$ In addition to the options for rule entrenchment discussed in a preceding subsection, other mechanisms for making a constitution more rigid or flexible exist in practice. Amendments can require more than one successful passage, a requirement designed to increase the salience of the issue to any stakeholders who might favor or oppose the amendment. The assent of subsidiary authorities can also be required, as is the case with the United States; Article V of the US Constitution requires the assent of two-thirds of the states to ratify any proposed changes to the constitution. See Sanford Levinson, RESPONDING TO IMPERFECTION: THE THEORY AND PRACTICE OF CONSTITUTIONAL AMENDMENT, 110-134 (1995). Finally, separable amendment thresholds can be used to identify areas of the constitution subject to higher barriers to amendment, up to and including making certain aspects of the constitution unamendable altogether. See Albert, supra note 14, at $247-51$.

${ }^{27}$ Nathalie Behnke \& Arthur Benz, The Politics of Constitutional Change Between Reform and Evolution, 39 J. FEDERALISM 213, 213 (2009).

${ }^{28}$ For example, the comparative flexibility of constitutions has been linked to increases in constitutional endurance. See Zachary Elkins, Tom Ginsburg, \& James Melton, The ENDURANCE of NATiONAL CONSTITUTIONS (2009).

${ }^{29}$ However, the nature of constitutional jurisprudence provides an additional outlet by which constitutional change can occur: instead of amending the constitution, changing judicial
} 
offs, constitutional scholars have focused on identifiable effects from different amendment procedures, ${ }^{30}$ in addition to the rate of change that more or fewer barriers to amendment imply. ${ }^{31}$

A broader characterization of the institutional design trade-offs implicit to the choice of amendment rules surrounds the need for a constitution to adapt to new developments while entrenching the system from self-interested behavior, ${ }^{32}$ at some point, amendment rules that are too flexible would mean none of the rules of the game are insulated from political pressures. ${ }^{33}$ The need for constitutional change has also been linked to political change and the dysfunctional performance of the existing constitutional regime. ${ }^{34}$ Each of these insights displays the fact that amendment procedures (and the frequency of amendment the procedures create) exist in a complex dynamic with the ongoing operation of the political system in both prior and subsequent periods. This is no different in the case of blockchainsthe rules for changing the underlying fundamental rule set are implicated in complex ways in the processes of governance and cryptocurrency output. In particular, constituents of blockchains face low exit costs, which provides an alternative to both enduring a rule set that is too rigid to change, as well as weathering changes of a greater frequency and magnitude than they would prefer. ${ }^{35}$

interpretations of constitutional requirements can adjust the constitution to reflect changing social conditions. See id.

${ }^{30}$ One such trade-off focuses on the extent to which the constitution includes high levels of detail as creating less need for downstream judicial interpretation. See Albert, supra note 14. Depending on the rigidity of the amendment rule, judicial interpretation may achieve a high level of finality. In the United States, for example, only four Supreme Court rulings had been reversed by constitutional amendment by 1988. See, e.g., Louis Fisher, CONSTITUTIONAL DiALOGUES: InTERPRETATION AS POLITICAL PROCESS 201 (Princeton University Press, 1988).

${ }^{31}$ This insight has been developed empirically, for several scholars have linked the length and detail of constitution to the frequency of amendment. See, e.g., Gabriel Negretto, Replacing and Amending Constitutions: The Logic of Constitutional Change in Latin America, 46 Law \& Soc'y Rev 749-79 (2012); Elkins et al., supra note 28.

32 See Tom Ginsburg \& Eric A. Posner, Subconstitutionalism, 62 Stan. L. Rev. 1584-626 (2010).

${ }^{33}$ A constitutional scholar who views constitutions as a bargain amongst powerful government actors (e.g., a legislature and a monarch or executive, typically) equates strengthening amendment rules with facilitating minority rights and the rule of law, emphasizing the role of stability as a crucial input to the rule of law. See Roger D. Congleton, Perfecting Parliament: CONSTITUTIONAL REFORM, LIBERALISM, AND THE RISE OF WESTERN DEMOCRACY 287 (Cambridge University Press, 2010).

${ }^{34}$ For example, the extent to which a given political system displays fragmentation of parties has been shown to interact with the nature of amendment procedures and the frequency. Gabriel Negretto, Replacing and Amending Constitutions: The Logic of Constitutional Change in Latin America, 46 LAW \& SOC'Y REV (2012); see also Negretto, supra note 31, and Schofield, supra note 18.

${ }^{35}$ Frequency of changes to a given blockchain network's rule set is already a margin defining distinct cryptocurrencies. Ethereum has changed its rules much more frequently than Bitcoin. See Primavera 
Furthermore, an important distinction exists in the literature between amending the constitution and amending the amendment procedures contained therein. A stable amendment procedure is a key measure of constitutional durability. ${ }^{36}$ Changes to the constitution are expected, but changes to the means by which the constitution can be changed signal a very different kind of change. This is related to the fact that amended rules have been characterized as directly expressing fundamental constitutional values. ${ }^{37}$ Similarly, amendment processes themselves can be understood as having a revelatory function, whereby the basis for the legitimacy of governance is revealed through both proposed and successful constitutional amendments. ${ }^{38}$ Changing the means of amendment is thus more of a meta-amendment, as compared to the more common changes to the underlying structure, and may indicate that such a change implicates much more fundamental changes to governance than other amendments. ${ }^{39}$ Different changes to cryptocurrency blockchains can be understood as implicating these concerns to very different degrees, as the discussion in section $\mathrm{V}$ emphasizes - some changes to blockchains are the equivalent of ordinary amendments, whereas others are the equivalent of changing amendment rules themselves.

Constitutional design writ large is seen as a trade-off between the economies of scale in governance that a single fundamental rule set creates and the agency costs associated with the necessarily representative government that governance at such a scale entails. ${ }^{40}$ One of the fundamental challenges in constitutional design is to restrain the lawmaking power once this power has been vested in representative agents who are only periodically and at best imperfectly disciplined by the constituents whose governance preferences their choices ideally represent. ${ }^{41}$ Of course, there is a dynamic element to these design choices, which implies that the fundamental ruleset should, to the extent possible, minimize future transaction costs associated with governance to reduce the need for costly constitutional change. ${ }^{42}$ The study of constitutions for the design of fundamental

de Filippi \& Aaron Wright, Blockchain And The Law: The Rule of Code 188 (Harvard University Press, 2018).

${ }^{36}$ Rasch \& Congleton, supra note 8.

${ }^{37}$ See Albert, supra note 14.

${ }^{38}$ Richard Albert, Nonconstitutional Amendments, 22 CAN. J. L. \& JURISPRUDENCE 5-47 (2009).

${ }^{39}$ Not all changes to amendment procedures are necessarily so fundamental; Sweden's changes to its amendment procedures were a necessary consequence of reducing the number of chambers of the legislature, as opposed to being directly intended to significantly change the way the constitution could be changed in the future. See Congleton, supra note 33.

${ }^{40}$ Ginsburg \& Posner, supra note 32, at 1594-97.

${ }^{41}$ Friedrich A. Hayek, The ConStitution of Liberty, 183-88 (1960).

${ }^{42}$ Riley, supra note 5, at 163-67. 
rule sets concludes that these sets of rules trade off between facilitating governance at scale (as well as the underlying processes being governed) ${ }^{43}$ and creating principal-agent problems on the part of those individuals who directly take part in governance. In the case of permissionless blockchains, the network participants who process and validate cryptocurrency transactions are the equivalent of representative agents that are only constrained by the network rules and the choice of users to transact and store value in one cryptocurrency or another. I explain this analogy in detail in the following section.

\section{BLOCKCHAINS AND GOVERNANCE}

Blockchains are, at their most fundamental, a ledger distributed across numerous networked computers that share a known process for updating entries to the shared ledger. The blockchains supporting most major cryptocurrencies share two important definitional components: (i) changes to the underlying ledger update original entries in discrete blocks of information, ${ }^{44}$ as opposed to overwriting them; and (ii) these changes to the ledger occur via a decentralized process among participants on a particular blockchain network. ${ }^{45}$ The way in which decentralized updates to the common ledger occur is defined by the consensus algorithm put in place by a given blockchain. This consensus algorithm (the rules for determining how changes to the ledger occur) is part of the initial conditions that are coded into the architecture of the blockchain itself. In what is perhaps the most famous blockchain - that underlying Bitcoin - the consensus algorithm creates a race

\footnotetext{
${ }^{43}$ See AlSton et al., supra note 18, at 307-16, for a discussion of law (including constitutional law) as a scalar mechanism facilitating social interactions and transactions.

${ }^{44} \mathrm{~A}$ blockchain is literally a chain of blocks of information (or code) referencing earlier states in the ledger duplicated across numerous network nodes. Luke Fortney, Blockchain Explained, INVESTOPEDIA (updated Nov. 26, 2019), https://www.investopedia.com/terms/b/blockchain.asp, [https://perma.cc/GFV7-BKHG].

${ }^{45}$ A common misconception is that all blockchains use decentralized "permissionless" governance. In reality, many of the commercial applications already in use utilize permissioned blockchains, where a central authority grants permission to certain network nodes to make specific alterations to the underlying common ledger. Supply chain innovations utilizing blockchain stand as a prominent example of this. All blockchains are a form of distributed ledger duplicated across numerous network nodes that can only be updated as opposed to amended, but only some blockchains are permissionless. See Rainer Böhme et al., Bitcoin: Economics, Technology, and Governance, 29(2) J. Econ. Persp. 213, 213-38 (2015) for a discussion of blockchain's definitional properties; see also Kevin Werbach, The Blockchain and the New Architecture of Trust, 58-63 (2018).
} 
among network nodes ("participants") ${ }^{46}$ to solve a cryptographic hash function, ${ }^{47}$ a method that, while largely secure against fraudulent attempts to update the ledger to reflect Bitcoins that do not exist, ${ }^{48}$ is graphics processor-intensive and so consumes considerable amounts of energy. ${ }^{49}$ This particular consensus algorithm, known as proof-of-work ("PoW"), operates in a winner-take-all fashion-when one network participant has successfully solved the cryptographic puzzle, the rest of the nodes that were processing potential solutions to the hash function are effectively back to square one. From the perspective of these miners, the energy expended in processing network users' transactions receives no reward until the next set of proposed changes to the Bitcoin ledger is accepted through the next successful participant's resolution of the hash function. ${ }^{50}$ Because each network participant's processor is engaged in an electrically costly race to solve the cryptographic puzzle, this method of achieving consensus over proposed changes to the network ledger is called "proof-of-work;" a successfully proposed block represents proof of energy expended to validate network transactions. To the layperson, this means processing transactions on the Bitcoin network is very electricity-intensive, and only more so as the value of Bitcoin increases and more participants compete to receive a fixed

\footnotetext{
${ }^{46}$ Network nodes are often referred to as miners because their incentives to process transactions are created by a reward of new units of the cryptocurrency. Throughout this piece I will refer to the individuals in control of these nodes as network participants, because of their role in the production, processing, and verifying of cryptocurrency transactions. Individuals who use cryptocurrency to transact or store value are instead network users, because they play no direct role in the maintenance of the network (unless they are also a network participant).

${ }^{47}$ A cryptographic hash function is basically a puzzle where network participants race to guess an unknown answer to a unique puzzle (typically a string of numbers or characters preceded by a given number of zeros when resolved by the hash function). The Ethereum blockchain currently relies on a similar consensus method, although an upcoming change to this consensus method is discussed in section V. BlockchaIN SuPER FAQ, ConSENSyS, https://consensys.net/knowledge-base/blockchainsuper-faq/ [https://perma.cc/PD7N-WPEB].

${ }^{48}$ Proof-of-work consensus algorithms have one weakness associated with network processing power. If over 51 percent of the computing power on the network at any given time can be controlled by one entity, then the ledger can be updated to reflect transactions that would be at odds with network rules. This creates the possibility for double-spending Bitcoins. In practice, however, the major cryptocurrencies tend to have sufficient network participants to where a 51 percent attack is unlikely. See, e.g., Werbach, supra note 45 , at 100. Less widely adopted cryptocurrencies (with accordingly lower numbers of network nodes) have suffered from 51 percent attacks, however. See Jordan French, Ethereum Classic's "51\% Attack," \$1 Million Loss, Raise Concerns About Security, THE STREET (Jan. 14, 2019), https://www.thestreet.com/investing/bitcoin/attack-against-ethereumclassic-14832327 [https://perma.cc/H2ME-75NQ].

${ }^{49}$ See generally Arvind Narayanan et. al., Bitcoin and Cryptocurrency Technologies: a Comprehensive Introduction (2016); see also Harald Vranke, Sustainability of Bitcoin and Blockchains, 28 CURRENT OPINION IN ENVTL. SUSTAINABILITY 1, 1-9 (2017).

${ }^{50}$ See Zibin Zheng et al., An Overview of Blockchain Technology: Architecture, Consensus, and Future Trends, 6 IEEE Int'l Cong. on Big Data 557, 560 (2017).
} 
amount of Bitcoins for each successful resolution of the hash function. ${ }^{51}$ Because anyone who has a sufficiently powerful computer, internet access, and electricity can become a network participant, this makes these types of blockchains permissionless, meaning no central authority controls who can make changes to the network ledger or participate in network governance.

What the choice of the PoW consensus algorithm has done for Bitcoin is to drive a wedge between its initially proposed dual functions as a store of value and a payment network. Bitcoin's growing salience to potential adopters has made it an increasingly successful store of value (with the exception of the price volatility ${ }^{52}$ associated with speculative interest and regulatory interventions in a variety of countries), but this increase in value has created problems for Bitcoin's ability to serve as a competitive payment network, given the cost and time required to process a single transaction. This has led to considerable debate within the Bitcoin community itself surrounding how to overcome these problems that are a direct result of the initial choice of consensus algorithm. ${ }^{53}$ In the case of Bitcoin, changes to the rules governing the blockchain network's processes occur through a fork in the blockchain itself, ${ }^{54}$ which is successful if a majority of other network nodes accept the new code as changed, as compared to the original code. ${ }^{55}$ This has led to difficulties in successfully changing the underlying architecture of the network. While this is not to say a given proposed fork in the Bitcoin blockchain should or should not have occurred, a wide variety of network participants have expressed frustration at the current means of changing the rules of the blockchain itself, which emphasizes the importance of blockchain governance.

The process described regarding the simultaneous processing and validation of a set of network transactions, coupled with the reward of new units of

\footnotetext{
${ }^{51}$ See Christopher Malmo, One Bitcoin Transaction Consumes as Much Energy as Your House Uses in a Week, VICE: MOTHERBOARD (Nov. 1, 2017), https://motherboard.vice.com/en_us/article/ywbbpm/bitcoin-mining-electricity-consumptionethereum-energy-climate-change [https://perma.cc/876Z-8L72].

${ }^{52}$ Tony Klein et al., Bitcoin Is Not the New Gold-A Comparison of Volatility, Correlation, and Portfolio Performance, 59 InT'L REV. OF Fin. ANALYsis 105, 115 (2018).

53 See Oscar Nilsson \& Dorna Garagol, Public Blockchain Communities: A Study on How Governance Mechanisms Are Expressed within Blockchain Communities, 3-4 (2018) (unpublished M.A. thesis, University of Gothenburg) (on file with the University of Gothenburg Department of Applied Information Technology).

${ }^{54}$ A blockchain fork is when changes to the code are adopted by a subset of network participants, while other participants reject these same changes, resulting in two distinct blockchains moving forward from the point of rule disagreement. The following section discusses the predominant types of forks and their implications for governance, in greater detail.

${ }^{55}$ Bruno Biais et al., The Blockchain Folk Theorem, 14 (Toulouse Sch. of Econ., Working Paper No. 17-817, 2018).
} 
cryptocurrency for doing so, creates a set of margins that together define the incentives of network participants. The margins of cryptocurrency reward amount, network fees ${ }^{56}$ block size, and puzzle difficulty together to create an equilibrium expected payoff as a function of the energy expended by a given network participant to successfully resolve a set of proposed transactions on the network. ${ }^{57}$ The greater the anticipated reward (increasing as puzzle difficulty decreases and rewards and fees increase), the greater the number of network participants likely to expend the energy in racing to solve the underlying hash function. Thus, one significant area of debate among network participants is how to calibrate these factors to the benefit of all participants. Of course, the nature of processing transactions is energy-intensive, which means energy prices greatly determine where network participants are physically located, ${ }^{58}$ as well as these participants' relative positions with respect to proposed changes to the rules governing the blockchain. Importantly, though, a final definitional component of participants' incentives is that of the costs of exit - to the extent that changes to their incentives on one blockchain are sufficiently undesirable, permissionless blockchain participants can (at some cost) opt into a different cryptocurrency blockchain that better matches their governance preferences.

This all suggests that even in the case of cryptocurrency networks, for which some of the comparative benefits depend in great part on the blockchain's immutability, the governance of system-wide processes (often tightly tied to the consensus algorithm by which changes to the ledger occur) and the process by which the rules of this governance can change, have significant implications for the intended outputs of the network itself. Put differently, the rules about making or changing rules governing a blockchain matter fundamentally. This insight comes as no surprise to any legal or constitutional theorist, of course. In addition to the Bitcoin community, other cryptocurrency blockchains (e.g., Ethereum) are confronting similar problems in scaling informational changes on the network and are instead proceeding with a different set of proposed changes, with the intent of changing the underlying consensus algorithm itself. Ethereum has emerged as a competitor to Bitcoin among major cryptocurrencies and is currently testing a

\footnotetext{
${ }^{56}$ In addition to the rewards of new cryptocurrency units, network participants receive a set fee for every transaction they process. In the case of cryptocurrencies like Bitcoin that have a known upper limit to the amount of coins that will circulate, it is expected that these fees will prove sufficient to incentivize network participants once new coin rewards have been exhausted.

57 See Joshua A. Kroll et al., The Economics of Bitcoin Mining, or Bitcoin in the Presence of Adversaries, (Workshop on the Econ. of Info. Sec., Washington, D.C.), June 11, 2013, at 1, 4-5; see also NARAYANAN et al., supra note 49, at 108.

58 See Paul Roberts, This Is What Happens When Bitcoin Miners Take Over Your Town, PoLITICO (Mar./Apr., 2018), https:/www.politico.com/magazine/story/2018/03/09/bitcoin-mining-energyprices-smalltown-feature-217230.
} 
different process by which rules as fundamental as the underlying consensus algorithm are changed. This provides a clear example by which dominant cryptocurrencies are currently competing in terms of governance-a phenomenon discussed in detail in sections $\mathrm{V}$ and VI.

This concern over governance is unique to permissionless blockchains. In the case of a central authority tightly defining the location or powers of a particular network node, the means by which rule changes occur are not necessarily related to the network nodes' roles or preferences in any way and instead are a function of ordinary centralized firm decision-making. ${ }^{59}$ In contrast, the design of permissionless blockchains means anyone with the right hardware, internet access, and reliable electricity can become a participant and hence have incentives and beliefs about the appropriate scope and form of blockchain governance. Decentralized validation of network processes coupled with distributed maintenance of the underlying ledger means that network participants necessarily have direct influence over the continuance or change of the ruleset governing all network processes. ${ }^{60}$ This implicates an aspect of governance typically reserved to public organizations, where constituents cannot opt out of the processes to which they are subject. Arguably, participants on a given blockchain are facing much lower exit costs in terms of their preferred governance than an employee subject to the centralized policies determined by private firm leadership. Their decision is more akin to investors who choose to move money from one firm to another based upon the governance decisions made by the firms in question. Nonetheless, on permissionless blockchains, the validation of network transactions is designed to prevent participants from evading the universal rules. This makes a similar influence over input to the ruleset incentive compatible. Unlike traditional firms, the equivalent of shareholders in a given permissionless blockchain are also engaged in the output of that blockchain. This stands as a powerful departure from traditional models of private governance. ${ }^{61}$

Similarly, blockchain network participants, and to a lesser extent, users, also have strong beliefs about the appropriate scope and form of the functions to which a given blockchain will be applied. Thus, certain proposals to alter the fundamental rule set underlying a given blockchain are frequently argued to be at odds with the "constitutional" principles that guided the definition of the blockchain in the first

\footnotetext{
${ }^{59}$ DE FILIPPI \& WRIGHT, supra note 35 , at 31.

${ }^{60}$ Zheng et al., supra note 50.

${ }^{61}$ See Nick Cowen, Markets for Rules: The Promise and Peril of Blockchain Distributed Governance 9(2) J. ENTR. \& PUB POL'y 213 (2019).
} 
place. ${ }^{62}$ Originalist arguments have appeared in the context of proposed changes to the Bitcoin blockchain, and in the case of Ethereum, one of the founders plays a major role in ongoing debates about changes to the underlying blockchain. ${ }^{63}$ Debates surrounding the intent for Bitcoin to become a major store of value and payment network, as compared to the intent for the currency to be immutable in terms of the rules governing its creation and transfer, are fundamentally debates about the true constitutional spirit of the blockchain as expressed through the specific rules governing network processes.

\section{A. Blockchains and Constitutions}

How, then, can blockchains be understood as a system of governance analogous to that created by a constitution? The participants, or network nodes, in a given blockchain play the role of the government, whereas the users of a given blockchain can be seen as constituents. While network participants are not representative agents in the exact sense politicians are, they are performing governance functions on behalf of users who request transactions or store value on the cryptocurrency blockchain. The fundamental rules of the blockchain create a form of agency control by constraining the participants in the interest of the users, which creates an equilibrium that operates to the benefit of the constrained participants. Changes to cryptocurrency blockchains can thus affect any of the following: (i) the core definition of the underlying unit of value; (ii) the process of transactional validation by network participants; (iii) the incentives of network participants (often implicated by changes to transactional processes); or (iv) the comparative ability of a given blockchain to achieve its network objectives in comparison to the ability of competing blockchains to do so.

An important distinction between blockchain governance and modern constitutional democracies lies in the absolute lack of separation of powers. Blockchain participants exercise executive, legislative, and judicial functions simultaneously. As participants successfully solve the cryptographic hash function underlying the proof of work algorithm, they are simultaneously processing network transactions and generating network resources (the underlying

${ }^{62}$ See, e.g., Shruti Rajagopalan, Blockchain and Buchanan: Code as Constitution, in JAMES M. Buchanan: A Theorist of Political Economy and Social Philosophy 19-20 (Richard E. Wagner ed., Palgrave Macmillan, 2019) (detailing Bitcoin participants' conflicting views on a proposed change to the code that would eliminate the cap on the number of transactions that could be processed by the network in a given time frame).

${ }^{63}$ Vitalik Buterin regularly discusses proposed governance changes publicly. See, e.g., Vitalik Buterin, Notes on Blockchain Governance, HACKER NOON (May 30, 2019), https://hackernoon.com/notes-on-blockchain-governance -ob65o3pod [https://perma.cc/GE4VA385]. 
cryptocurrency), while other participants mechanically validate proposed transactions' conformity with underlying network rules. ${ }^{64}$ Each of these processes can be likened to a distinct role of government. Cryptocurrency production and the proposal of minor rule changes, together, are the equivalent of legislation. Processing transactions of existing cryptocurrency is like the execution of law. Ensuring conformity with network rules is akin to the judicial function of finally determining violations of the law by constituents. ${ }^{65}$ Currently, the incentives to perform these functions are linked to the production function; miners engage in costly processing of transactions due to the anticipated reward for successfully solving the underlying cryptographic hash function that accompanies their doing so.

Blockchains, unlike government processes and judicial review, do not allow for the informal remedies for adjustments to the rule structure to better fit the needs and aims of those governed by the ruleset. Granted, blockchain participants can adjust their uses of the blockchain to some limited extent, but they cannot directly interpret the underlying fundamental rule structure like government and judicial actors can in terms of constitutional implementation and interpretation. Put differently, the extent of discretion afforded to the government to make the Civil Rights Act a reality, or the extent of judicial interpretation that the Bill of Rights has required (and will continue to require), far exceeds the scope of choice blockchain network participants have in terms of the scope, form, and frequency of transactions to undertake. Coherence and supremacy are practically guaranteed in blockchain processes, which can be a double-edged sword when it comes to punishing bad-faith actors on the network, as the subsequent discussion about the DAO hack on the Ethereum blockchain emphasizes. Reversing a bad action under current governance processes takes the equivalent of a constitutional amendment.

One of the most important distinctions between changes to blockchains and constitutional amendment processes surrounds the notion of forking the chain of code. Three distinct, predominant means ${ }^{66}$ of forking exist: (i) a soft fork, which results in compatibility for network nodes that have not yet adopted the new rule change; (ii) a hard fork that would result in network nodes that have not accepted

\footnotetext{
${ }^{64}$ Narayanan et al., supra note 49.

${ }^{65}$ For an extensive discussion of the relevance of blockchains to traditional legal processes see De Filippi \& Wright, supra note 35.

${ }^{66}$ A number of distinctions define how hard forks can proceed, which results in at least eight distinct types of hard forks. Nonetheless, for the purposes of this analysis focused on governance and exit costs, the key distinction is a hard fork that results in two viable chains, and one that does not. The former presents an exit option for network participants in a way that the latter does not. See, e.g., Sean Stella, What is a Hard Fork? Hard Forking Explained!, HARDFORKInG.COM, https://www.hardforking.com/what-is-a-hard-fork/.
} 
the new rule change rejecting blocks created under the new rule set; and (iii) hard forks that result in two permanently separate versions of the underlying blockchain, one under the old ruleset and one under the new ruleset. ${ }^{67}$ Major rule changes, including updates to the block size, result in hard forks, which create the possibility for deterioration in network processes and outright errors, and as such, involve considerable debate in a given blockchain community prior to their adoption. In the second case of hard forks, unlike constitutional amendment, two blockchains can exist where there once was one, and participants and users subsequently decide (through their support of network processes and choice of transactional medium) which chain to support. ${ }^{68}$ These choices in rule updates each influence the exit costs facing participants and users if they do not like a particular rule change. ${ }^{69}$ With both soft and hard forks that do not result in a new cryptocurrency, the participants and users can only exit from the underlying blockchain entirely, as opposed to continuing their participation and use on the chain of the forked cryptocurrency that they most prefer. The choice of whether or not a hard fork will result in a viable second chain (and associated currency) is clearly utilized in cryptocurrency communities, with an announced hard fork of Ethereum (Constantinople) that will not result in a viable second chain, accompanied by discussion as to whether a second chain would emerge. ${ }^{70}$ Such an outcome depends on the extent to which participants accept or reject the proposed rule changes, as well as the specific changes to the protocol layer itself that the proposed update makes.

In contrast, after a constitutional amendment, constituents are uniformly governed by the altered constitution, and the old constitution only governs those legal claims that emanated when the prior version of the constitution was in force. This means fundamental changes in rule sets on blockchains are subject to competitive pressures after the fact, with the former rule set potentially remaining a viable transaction and currency network, which allows for subsequent revelation of the perceived value of the network change. This distinction means blockchains are subject to competitive pressures on a number of margins which constitutional governance is not, notwithstanding the limited ability of constituents to move from

\footnotetext{
${ }^{67}$ See De Filippi \& Wright, supra note 35, at 24; Narayanan et al. supra note 49, at 73-75; Noelle Acheson, Hard Fork vs. Soft Fork, COINDESK (Mar. 16, 2018), https://www.coindesk.com/information/hard-fork-vs-soft-fork/.

${ }^{68}$ See Biais et al., supra note 55; see also Arruñada, Benito, \& Luis Garicano, Blockchain: The birth of decentralized governance (May 11, 2018) (unpublished manuscript) (on file with Pompeu Fabra University Economics and Business Working Paper Series).

${ }^{69}$ Alastair Berg \& Chris Berg, Exit, voice and Forking (2017) (unpublished manuscript), available at: https://papers.ssrn.com/sol3/papers.cfm?abstract_id=3081291.

${ }^{70}$ Rachel Rose O'Leary, What to Expect When Ethereum's Constantinople Hard Fork Happens, COINDESK (Jan. 11, 2019), https://www.coindesk.com/what-to-expect-when-ethereumsconstantinople-hard-fork-happens.
} 
one constitutional regime to another (to the extent they can afford to do so). This also means that in cases where a cryptocurrency forks to reflect distinct governance preferences on the part of different groups of network participants, this operates as a low-cost form of exit - both groups can be governed by their preferred set of constitutional rules, although they can no longer transact with one another on the same blockchain as before. ${ }^{71}$ All of this implies that the choice to fork presents an important strategic margin for blockchain network participants considering rule changes. ${ }^{72}$

The scope of activities governed by a blockchain is much smaller than those defined by the legislative process in modern constitutional orders; the former is limited to a small number of processes, whereas the latter is only cabined by the boundaries the constitution places on the action of the government. This variance in "legislative" scope has a corollary implication: the comparative importance of the judicial function increases as legislative scope increases. If a governance regime has a known and limited set of functions, the interpretation of the conformity of these functions with underlying network rules is a relatively simple process. However, as the nature and interpretability of network functions increases, the challenge, and hence, systemic importance of ensuring that these functions are in accordance with underlying rules similarly increases. This results in a comparative certainty of application of blockchain rules, as compared to constitutional rules; although, to the extent that blockchains follow through with proposed changes to include decentralized transactional processing and validation, this is likely to create unique challenges, as discussed in the section treating the Lightning Network and Sharding below. This means the implementation of changes to the underlying blockchain rules is much more certain. Blockchain rule changes have less need for interpretation in subsequent periods but are also less subject to downstream political pressures than constitutions, which require executive discretion in their implementation, coupled with judicial interpretation to oversee the constitutionality of this implementation.

Unlike constitutional government at the national level, those governed by a particular blockchain face much lower exit costs and can also be simultaneously governed by several blockchains to the extent they mine or transact in more than one cryptocurrency. ${ }^{73}$ This provides an interesting analog to the decentralized

\footnotetext{
${ }^{71}$ Narayanan et al., supra note 49, at 171-73.

72 Alastair Berg, Chris Berg \& Mikayla Novak, Blockchains and Constitutional Catallaxy, (Dec. 4, 2018) (unpublished manuscript), available at: https://papers.ssrn.com/sol3/papers.cfm?abstract_id=3295477.

${ }^{73}$ Cowen, supra note 61.
} 
governance arrangements studied in a number of contexts. ${ }^{74}$ Consider the extent to which blockchain participants and users can freely enter and exit the governance arrangements of a given cryptocurrency, and compare it to the proposed and existing real-world governance schemes intended to harness similar governance benefits as a function of the largely voluntary presence of their constituents; there is significant overlap between the two. ${ }^{75}$ The comparatively low-cost exit option both disciplines blockchain network participants in their choices of rule changes and serves as a margin of competition when rule changes implicate exit costs directly, as the discussion in section VI emphasizes.

The means by which constitutional amendment can proceed in ordinary political systems provide a blueprint by which to consider similarly fundamental governance changes on permissionless blockchains. Ultimately, the certainty of the application of blockchain network rules and the comparatively low exit costs facing cryptocurrency users reduce the importance of ex-post constitutional interpretation as amending the ruleset itself. For example, in the case of judicial review, there is no clear analogy because of the decentralized fashion in which network transactions are validated; absent an ex-ante agreement among over half of network participants, a given network node (run by a network participant) could not use its own discretion to validate transactions at odds with the rules of the blockchain. ${ }^{76}$ The next informal means of constitutional change is that of adjustments in government processes and norms; in the case of blockchains, this involves participants and users' understanding of network capabilities and limitations, and their ability to adjust their network uses accordingly. One example of this would be users limiting their transactions on the Bitcoin network to a certain minimal threshold, below which the network fees for doing so do not make economic sense. Next, it is possible in theory to develop subsidiary layers of code governed by distinct rule sets (or simply rules that do not need to be validated by the entire network); in each case, these would operate like political and administrative decentralization, respectively. In the case of distinct rule sets, different network nodes (or a set of nodes) would operate their own rules or processes, although this would create coordination costs in reconciling different underlying processes with the primary chain itself; this is

\footnotetext{
${ }^{74}$ Tom W. Bell, Special International Zones in Practice and Theory, 21 CHAP. L. REV. 273 (2018). From early Chinese special economic zones to more sophisticated decentralization arrangements, there has been an increasing recognition of the value of competitive governance in facilitating more effective or representative institutions, as well as the political economy challenges associated with doing so. See, e.g., Lotta Moberg, The Political Economy of Special Economic Zones, 11 J. of Institutional ECON. 167-90 (2015); Lotta Moberg, The Political EConomy OF Special ECONOMic Zones: CONCENTRATING ECONOMIC DEVElopMENT (Taylor \& Francis, 2017).

${ }^{75}$ Mark Frazier, Emergence of a New Hanseatic League: How Special Economic Zones Will Reshape Global Governance, 21 CHAP. L. REV. 333 (2018).

${ }^{76}$ Narayanan et al., supra note 49.
} 
something the Ethereum blockchain may be better suited to facilitating, given its focus on more complex transactional processes. ${ }^{77}$ In the case of the same rules, a subset of network nodes would be responsible for processing and validating network processes without wholesale validation occurring across the entire network. Importantly, in order for these decentralized processes to occur, this would require the support of a sufficient majority of network participantsblockchain's equivalent of a constitutional amendment. Finally, in the most wellknown process (given the debates surrounding formal changes to the network rules), if a sufficient number of network nodes adopt a proposed rule change or changes, then the primary blockchain will be governed by these rules accordingly (with those set of network nodes that rejected the rule change operating under the old set of rules if a specific type of hard fork occurs). ${ }^{78}$

Accordingly, when it comes to governance, permissionless blockchain participants have three alternatives when it comes to adjusting governance. They can amend the rules underlying the blockchain itself (including those such as forking, which greatly shape exit costs), they can develop subsidiary governance processes that prevent the need for adaptation of the main blockchain, or they can adjust their use of a given blockchain, either directly, or by choosing to exit altogether and participate in or use another blockchain whose governance better resolves that participant's fundamental aims.

\section{B. Cryptocurrency Governance Debates}

Governance debates in cryptocurrency communities have resulted in a number of proposed and realized solutions that contain direct parallels to constitutional design choices intended to reduce agency costs while simultaneously capturing scale economies in governance. These debates have implicated fundamental beliefs as to the most important governance principles that should be realized in the rule sets of a given cryptocurrency blockchain, as in the case of Ethereum and Ethereum Classic. Smaller changes to these blockchains (like block size) have nonetheless resulted in sufficient disagreement to where distinct governance regimes have emerged, such as the hard fork between Bitcoin and Bitcoin Cash. Different forms of subsidiary governance solutions have emerged as proposals, including both administrative and judicial decentralization, as well as elements of relational contracting, in the cases of the Bitcoin Lightning Network and the proposed Ethereum changes of Plasma and sharding. Wholesale change to governance processes is also being explored in the case of Ethereum's proposal to transition their blockchain from a proof-of-work consensus method to a proof-of-

\footnotetext{
${ }^{77}$ Arruñada \& Garicano, supra note 68, at 3.

${ }^{78}$ De Filippi \& Wright, supra note 35, at 31; Biais et al., supra note 55.
} 
stake algorithm, in what would be the closest case to amending amendment rules themselves. Finally, new entrants have explicitly conditioned their competitive advantage on choices of blockchain governance, suggesting that permissionless blockchains will continue to be an important locus of innovation in private governance as participants and users exit from blockchains whose governance choices are less representative than others.

\section{Ethereum and Ethereum Classic}

In 2016, the Ethereum community was split between two governance alternatives in response to a well-publicized hack resulting in the loss of nearly $\$ 50$ million of Ether. The Ethereum community had developed an organization for signaling community confidence in proposed applications (decentralized apps or dApps) for use on the Ethereum blockchain. The Decentralized Autonomous Organization (DAO) - effectively a complex smart contract running on the Ethereum blockchain - was intended to serve as a means of funding different proposed applications using the decentralized input of individual users. Following a screening process accomplished via known network figureheads, users who had bought tokens in the DAO could then, in theory, allocate these tokens to projects, and once projects had reached a 20 percent token threshold for acceptance, they would be funded via the Ether held by the DAO. ${ }^{79}$ Taken at face value, the DAO presented a governance innovation in that the investment decisions would have involved human decision-making by screening projects ex-ante through existing reputation, social capital, and authority within the community. This presented the direct possibility that smart contracts can still involve elements of relational contracting.

However, a flaw in the code intended to prevent "bank runs" (which required Ether withdrawn from the DAO in response to approved applications to be held for 28 days), enabled hackers to capture $\$ 50$ million of Ether ${ }^{80}$ This flaw in the code resulted in a major governance challenge for network users. Maintaining fidelity to what were argued to be core principles governing the blockchain meant allowing the hackers to get away with $\$ 50$ million. If instead, the blockchain could be dialed back to the state immediately preceding the hack, then those who benefited from the coins that were withdrawn from the DAO would never have

79 David Siegel, Understanding the DAO Hack, CoINDESK (June 25, 2016), https://www.coindesk.com/understanding-dao-hack-journalists/.

${ }^{80}$ See Klint Finley, A \$50 Million Hack Just Showed That the DAO Was All Too Human, Wired, https://www.wired.com/2016/06/50-million-hack-just-showed-dao-human/ (last visited Feb. 1, 2020); see also Matthew Leising, The Ether Thief, BLoomberG, https://www.bloomberg.com/features/2017-the-ether-thief/ (last visited Feb. 1, 2020). 
received their tokens. This latter option violated what many network participants saw as hard principles governing the creation and transfer of Ether on the Ethereum blockchain - transfers of Ether, once completed according to network rules, were immutable and given that the blockchain had already updated in light of the funds transferred out of the DAO. These funds going to the individual (or individuals) who exploited the flaw in the DAO code was a scenario seen as preferable to going against accepted rules governing the transfer of Ether on the Ethereum blockchain. ${ }^{81}$ What came into conflict here was the extent to which participants believed faithfulness to the immutability of network processes should take precedence over punishing clearly wrongful activity - essentially a conflict of constitutional beliefs. As of July 20th, 2020, Ethereum's market capitalization of $\$ 26.3$ billion was over thirty-five times that of Ethereum Classic. ${ }^{82}$ This suggests that more network participants and users believed in the importance of punishing bad actors, especially when it came to convincing new cryptocurrency adoptees that subsidiary systems on the Ethereum network had the willingness and the ability to punish bad actors who exploit weaknesses in code.

\section{Bitcoin v. Bitcoin Cash}

One strain the existing Bitcoin blockchain has put on network participants and users is the ability to process payments in a timely fashion. The block size of the Bitcoin blockchain, along with the difficulty of the hash function miners are solving, effectively determines the number of transactions the network can process in a given time period. Transactions per second is the common measure among major payment networks like Visa, with the Visa network averaging 1,700 transactions per second (and an underlying capacity of 47,000 transactions per second) ${ }^{83}$ In contrast, the Bitcoin network can process roughly seven transactions per second. ${ }^{84}$ More problematically, as the number of users of Bitcoin increases, this number of transactions remains the same because of the fixed size of blocks of data that are added to the Bitcoin blockchain and the largely fixed-rate at which the hash function is solved. Put differently, more network participants have to get in line for a process that only serves one customer at a time and that takes roughly the

\footnotetext{
${ }^{81}$ Vitalik Buterin, CRITICAL UPDATE Re: DAO Vulnerability, Ethereum Blog (June 16, 2017), https://blog.ethereum.org/2016/06/17/critical-update-re-dao-vulnerability/.

${ }^{82}$ Top 100 Cryptocurrencies by Market Capitalization, https://www.coinmarketcap.com (last visited July 20, 2020).

${ }^{83}$ Manny Trillo, Stress Test Prepares VisaNet for the Most Wonderful Time of the Year, VIsA VIEwPoINTs (Oct. 10, 2013), http://www.visa.com/blogarchives/us/2013/10/10/stress-test-preparesvisanet-for-the-most-wonderful-time-of-the-year/.

${ }^{84}$ K. Croman et al., On Scaling Decentralized Blockchains, in Financial Cryptography and Data SECURITY 16 (2016).
} 
same time to conclude each time. Thus, the more people transacting in Bitcoin, the greater the delay each transacting party faces before their transaction can conclude.

The implications are clear when it comes to Bitcoin's ability to be a payment network that can effectively serve a greater number of users. As a result, one hard fork resulted in a distinct cryptocurrency called Bitcoin Cash. Bitcoin Cash uses similar network processes to those supporting Bitcoin but involves a larger block size, thus allowing for greater transactional speed. As of July 20th, 2020, Bitcoin Cash's market capitalization stood at $\$ 4.1$ billion, while that of Bitcoin stood at over forty times greater, at nearly $\$ 170$ billion. ${ }^{85}$ Notwithstanding the large disparity in valuation, Bitcoin Cash is currently the fifth largest cryptocurrency by market capitalization, which suggests a large number of participants and users saw considerable value in the underlying network changes. However, the nature of comparative market capitalizations poses the question of the extent to which competition among closely related blockchains will result in multiple viable currencies. A given currency's value as a medium of exchange depends on widespread adoption, which creates a clear long-term downside to multiple closely related currencies. Can they compete as effectively as entirely distinct cryptocurrencies whose brand could more clearly signal underlying distinctions in blockchain governance choices? Forking as a means of reducing exit costs for network participants is not without its own costs to the network and may not always be a desirable option.

\section{Lightning Network (BTC) \& Plasma/Sharding (ETH)}

Interestingly, both Bitcoin and Ethereum participants have begun to explore solutions from the realm of relational contracting and decentralized governance in order to overcome the problem of scale created by the proof-of-work algorithm underlying each blockchain. In the case of the Bitcoin blockchain, a proposed modification would effectively create a subsidiary network for handling transactions among network users who repeatedly transact with one another. This proposal, known as the Lightning Network, is in response to the same underlying problem that led to the fork between Bitcoin and Bitcoin Cash. The Lightning Network is a proposal to move transactions off-chain by relying on existing relationships among Bitcoin users. This would create a payment channel between users, effectively allowing a limitless number of transactions between two parties that have a payment channel between them. Once the parties have closed the channel, the resulting net balance between the two would be posted to the Bitcoin blockchain in a single transaction, significantly reducing the network load among

\footnotetext{
${ }^{85}$ Trillo, supra note 83.
} 
users that repeatedly transact with and (likely) know one another. The proposal also envisions numerous channels open among multiple users, intended to create a web effect in which many transactions could occur off-chain, requiring only a few transactions to finally post to the blockchain. ${ }^{86}$ Existing constraints have meant that microtransactions in Bitcoin were impractical, given the long wait times and network fees that could outstrip the value of the transaction. This led to third parties willing to intermediate microtransactions, but the existence of such intermediaries stands in contrast to the founding principles of a decentralized payment system intended to fundamentally disintermediate transactions. By instead relying on the existing repeated relationships among Bitcoin network users, this proposed change leverages the power of relational contracting to overcome the problem of scalability created by the fundamental underlying ruleset.

Given the similarities between the underlying blockchains' protocols supporting Bitcoin and Ether, Ethereum participants have also developed an analogous solution, making the validation of smart contracts occur on a sublayer, a proposed update to network rules called Plasma. Plasma is similar to the Lightning Network, except it involves the validation of contracts as opposed to the validation of transactions among repeat players. In each case, only a small proportion of the activity on the subsidiary layer appears on and is validated by the network governing the primary blockchain. ${ }^{87}$ Importantly though, this does not involve the uniform enforcement of formally encoded blockchain rules to be operative on the blockchain sublayer, unlike sharding, which will be discussed next. Thus, this form of governance solution is most akin to administrative or political decentralization, where a subset of participants wholly govern subsidiary processes with a new set of rules that they themselves define.

Another related governance solution explored by Ethereum participants is called sharding and is incorporated into recent proposals made by Ethereum founder Vitalik Buterin. These proposals address the amount of policy adaptation likely to take place on the second layer of protocol, as opposed to the primary layer of the Ethereum blockchain itself. ${ }^{88}$ The proposed change is similar to classic

\footnotetext{
${ }^{86}$ See Joseph Poon \& Thaddeus Drvia, The Bitcoin Lightning Network: Scalable Off-Chain Instant Payments 1, 14 (Jan. 14, 2016) (draft ver. 0.5.9.2) (available at https://lightning.network/lightningnetwork-paper.pdf).

${ }^{87}$ See generally Joseph Poon \& Vitalik Buterin, Plasma: Scalable Autonomous Smart Contracts (Aug. 11, 2017) (working draft) (available at https://plasma.io/plasma-deprecated.pdf).

${ }^{88}$ Interestingly, Buterin noted that constant change to the blockchain's primary rule set would either result in considerable uncertainty for network participants or would require a high degree of centralized control — both are at odds with the fundamental beliefs surrounding stable decentralized governance that animated the creation of the Ethereum blockchain. See Vitalik Buterin, Layer 1
} 
governance solutions intended to overcome problems created by the increase in the scale of processes, whether it be from the scope of government policies, the number of people governed by them, or both. Sharding involves the replication of some or all Ethereum blockchain processes in a subsidiary blockchain managed by one or more network participants. ${ }^{89}$ The Ethereum logo is a crystal, which clarifies the governance design underlying sharding. Each fragment of a larger crystal, known as a shard, duplicates the internal molecular crystalline structure of the larger crystal. In practice, sharding is a blockchain-specific form of judicial decentralization, where the validation of network transactions occurs on only a subset of participants' nodes. Where this process stands as a potentially unique governance innovation is that judicial decentralization is typically a function of political decentralization, where subnational governments define law that is subsequently adjudicated at the same level; here the blockchain rules would remain the same, but only a subset of network participants would be responsible for ensuring conformity with those rules. ${ }^{90} \mathrm{~A}$ fundamental trade-off of decentralization is squarely apparent in the case of sharding. Increases in terms of the scalability of network processes are weighed against the additional costs of governance on each individual shard, as well as the coordination costs of reconciling individual shard states with respect to the primary layer blockchain. The ability to maintain a greater number of transactions with greater variance in applicable rules can be seen as a governance solution designed to reduce the incentives to exit the blockchain due to the greater likelihood that a given user and participants' governance preferences will be accommodated in the more relational or decentralized arrangements intended to result.

\section{4. $\quad$ Proof-of-Stake (ETH)}

One of the most fundamental governance changes being debated among cryptocurrency network participants surrounds transitioning Ethereum from a proof-of-work (PoW) to a proof-of-stake (PoS) consensus algorithm. This proposed change directly approaches the problem of electricity intensity and network congestion leading to high transaction fees and slow processing time, two of the major obstacles to any cryptocurrency network functioning at scale. For commercial actors to readily adopt cryptocurrency payment methods, they need

Should Be Innovative in the Short Term but Less in the Long Term, Vitalik Buterin's website (Aug. 26, 2018), https://vitalik.ca/general/2018/08/26/layer_1.html?ref=tokendaily.

${ }^{89}$ See Jordan Raul, How to Scale Ethereum: Sharding Explained, Меdium (Jan. 10, 2018), https://medium.com/prysmatic-labs/how-to-scale-ethereum-sharding-explained-ba2e283b7fce; see also Hsiao-Wei Wang, Ethereum Sharding: Overview and Finality, Меdium (Dec. 26, 2017), https://medium.com/@icebearhww/ethereum-sharding-and-finality-65248951f649.

${ }^{90} \mathrm{Id}$. 
assurance that payments will process quickly and will not cost the vendor a significant proportion of the underlying transaction. Perversely, buying a car with a cryptocurrency may currently present fewer obstacles than buying a latte with a given cryptocurrency.

Transitioning from PoW to PoS involves a wholesale change to the algorithm that produces, changes, and validates cryptocurrency ledger states. Instead of a race to solve a cryptographic hash function, network consensus about proposed changes to the underlying ledger is achieved through Ether holders staking a portion of their holdings to become a network validator. The intention behind the design derives directly from the concept of increased exit costs creating beneficial governance incentives; validators that have staked a significant amount of valuable Ether are much more likely to have the continued stability and viability of the network in mind when validating new proposed blocks. Successfully proposed blocks will reward their relevant stakeholders not only in terms of new Ether but also via the fees associated with the transactions contained in a given block. ${ }^{91}$ The proposed intent is to run the new blockchain on a test network for a certain period of time, which makes this a phased transition, with the network eventually transitioning at a known rate to a blockchain exclusively supported by $\mathrm{PoS}$ as opposed to PoW processes. Ethereum's co-founder, Vitalik Buterin, is clearly concerned about governance compatibilities created by the simultaneous existence of different consensus algorithms, as some of his technical output suggests. ${ }^{92}$

The phased transition reflects the profound change in network processes that changing the consensus algorithm would entail. Unlike adjusting the size of blocks, transaction fees, and new currency rewards, or even creating a subsidiary layer of governance, a change to the consensus algorithm is equivalent to changing the amendment rules of a constitution. Thus, the debates surrounding the change have been longer and more intense than debates over alterations in fees and hash function difficulty. If Ethereum transitions entirely to a PoS protocol, then all fundamental rule alterations in the future will proceed via a completely different governance process. Essentially, the new consensus mechanism is a proposal to change the entire structure of government for the Ethereum blockchain, and as such, stands as an amendment to the amendment processes themselves, if not a wholesale constitutional overhaul. If successful, it will be worth observing closely how other competing blockchains react to the benefits and costs revealed by a completely new

91 See Jon Choi, Ethereum Casper 101, Medium.com (Oct. 21, 2017), https://medium.com/@jonchoi/ethereum-casper-101-7a851a4fleb0 [https://perma.cc/C2W9L87L].

${ }^{2}$ See Vitalik Buterin, A Guide to 99\% Fault Tolerant Consensus, Vitalik.CA (Aug. 7, 2018), https://vitalik.ca/general/2018/08/07/99_fault_tolerant.html [https://perma.cc/C8FP-DC2T]. 
system of blockchain governance, especially in the case of blockchains like Tezos and EOS that have emerged in direct response to the governance tensions described thus far. However, the magnitude of change and the implications for all the network processes on the Ethereum blockchain have led to numerous missed deadlines associated with the launch of this change, which was originally rumored to be completed sometime in $2017 .{ }^{93}$ Given how central to the long-term viability of the Ethereum network the PoS upgrade has been argued to be, it will be interesting to see whether network participants choose to support the upgrade, create a second currency based on the Ethereum blockchain up to that point (separate from Ethereum Classic), or exit the system altogether.

\section{Emergent Governance Competitors: Tezos \& EOS}

This type of governance competition has already been occurring in the realm of cryptocurrencies, though. When Tezos was released in 2017, its initial coin offering (ICO) was one of the most successful to that point, raising \$232 million. ${ }^{94}$ The amount raised in an ICO can be seen as a version of an initial public offering, except governed by considerably less regulatory oversight. As investors have crowded into the space, there has been considerable fraud in terms of the extent to which companies issuing new coins actually have viable business models to support the bet in their business model that a purchase of an ICO typically implies. ${ }^{95}$ In the case of Tezos, the innovation surrounded blockchain governance processes themselves, a direct competitive response to the strains facing the dominant cryptocurrency blockchains supporting Bitcoin and Ether. Importantly, since the emergence of Tezos, Ethereum proposals have converged on a similar governance solution: that of PoS described above. Tezos allows for token holders to vote on proposed changes to the network's governance protocols - a node proposes a change along with a price associated with the cost they require to implement the change. ${ }^{96}$ In theory, this would allow for dynamic network pricing of governance innovations. As importantly, the emergence of Tezos in response to existing governance problems among the dominant cryptocurrencies in terms of scalability displays competitive private governance in practice. However, it should be noted

${ }^{93}$ See Amy Castor, Ethereum's Difficulty Bomb: All Smoke, No Fire?, CoInDESK (Apr. 8, 2017), https://www.coindesk.com/ethereums-difficulty-bomb-smoke-no-fire [https://perma.cc/EWR4HSP5].

${ }^{94}$ Omri Barsilay, Tezos' \$232 Million ICO May Just Be the Beginning, ForBes (July 16, 2017) [https://perma.cc/3GHZ-3RRJ].

${ }_{95}$ See Dirk A. Zetzche, Ross P. Buckley, Douglas W. Arner, \& Linus Föhr, The ICO Gold Rush: It's a Scam, It's a Bubble, It's a Super Challenge for Regulators (2018).

${ }^{96}$ Liam Johnson, Tezos (XTZ) Pros \& Cons - Self-Amending Cryptocurrency Blockchain, BITCOIN FOR BEGINNERS (Aug. 15, 2018), https://www.Bitcoinforbeginners.io/cryptocurrencyreviews/tezos-xtz-review/ [https://perma.cc/HLK4-36RN]. 
that Tezos has since faced profound governance issues in the real world, something that has held up its deployment and resulted in numerous lawsuits against the company. ${ }^{97}$ While network participants and users should pay close attention to the governance changes being wrought on the networks in which they are invested, this does not mean that choices regarding corporate structure and regulatory compliance under public constitutional structures no longer matter. ${ }^{98}$ This lesson is often forgotten amidst the exuberance that early cryptocurrency adopters have for radical decentralization.

EOS is a more recently emergent competitor to the Ethereum and Bitcoin blockchains. EOS, in direct response to the scalability issues that created the governance challenges described thus far, is based upon a delegated proof-of-stake system. Similar to Tezos, EOS was the most successful ICO of its time, raising several billion dollars in support, although the exact number remains subject to the volatility of Ether, the cryptocurrency in which the ICO was denominated. ${ }^{99}$ Interestingly, the founders have directly argued that this system is analogous to the governance of a republic, where representative agents oversee the processing and validation of network transactions. ${ }^{100}$ These agents are elected by the network community, presumably with some form of punishment in subsequent electoral rounds if their adherence to network rules and fundamental governance beliefs is revealed to be lacking. Instead of staking the network's currency, participants and users vote on representative agents, with their number of votes being proportionally equivalent to the size of their stake in the network. ${ }^{101}$ This method is designed to overcome another fundamental governance challenge not unique to blockchains: money in politics. In the case of Ethereum's proposed changes, the requirement of large Ether holdings in order to become a network validatory could in theory result in these participants validating proposed blocks due to self-interest, as opposed to those blocks' conformity with the underlying ruleset of the network. By allowing participants and users to aggregate their votes behind network participants that

\footnotetext{
${ }^{97}$ Thijs Maas, The Curious Tale of Tezos: From a $\$ 32$ MILLION ICO to 4 Class Action Lawsuits, HACKERNOON.COM (Apr. 6, 2018), https://hackernoon.com/the-curious-tale-of-tezos-from-a-232million-ico-to-4-class-action-lawsuits-6f411b7aad7e [https://perma.cc/3HPM-V4QE].

${ }^{98}$ See Eric Alston "Blockchain and the Law: Legality, Law-like Characteristics, and Legal Applications," in James Caton, ed., HANDBOOK ON BLOCKCHAIN AND CRYPTOCURRENCIES. (Forthcoming 2021).

${ }^{99}$ Kate Rooney, A Blockchain Start-up Just Raised \$4 Billion without a Live Product, CNBC (May 31, 2018), https://www.cnbc.com/2018/05/31/a-blockchain-start-up-just-raised-4-billion-withouta-live-product.html [https://perma.cc/35ZP-EGWP].

${ }^{100}$ See Aaron Stanley, EOS: Unpacking the Big Promises Behind a Possible Blockchain Contender, COINDESK.COM (June 25, 2017), https://www.coindesk.com/eos-unpacking-the-big-promisesbehind-a-possible-blockchain-contender/ [https://perma.cc/8XGB-LUFX].

${ }^{101}$ See Berg, Berg, \& Novak, supra note 72, at 10.
} 
those individuals believe will process and validate transactions in good faith, EOS's consensus algorithm is designed to mitigate the scale advantage that large holdings in the network are likely to create for certain Ethereum stakeholders. ${ }^{102}$ This is not to argue to that EOS' chosen governance mechanisms are superior, but instead to emphasize how they serve as an example of governance innovations occurring as blockchain networks distinguish themselves from one another on the institutional margins defined in this article.

In this section, I have provided a variety of examples of the ways that permissionless blockchains have already begun exploring institutional forms more traditionally associated with public constitutional ordering. In the following section, I explore additional constitutional design features that are likely to emerge as a result of their nearly ubiquitous role in public constitutional ordering.

\section{Competitive Constitutional Governance on Blockchains}

Despite the numerous differences between the comparatively narrow set of network processes determined by a blockchain's fundamental rule structure and the broad range of human behavior governed by constitutions, those seeking to better understand governance debates surrounding blockchains would do well to consider the long history of constitutions. ${ }^{103}$ Debates about the fundamental principles of governance inevitably implicate constitutional amendments, and the extent to which a given governance structure can accommodate changes desired by a sufficient number of constituents can directly determine how well that governance structure can achieve its intended functions. In a competitive world, where network users and participants bet on the viability of one blockchain over another, governance provides an important margin for competition. This effectively determines how well a blockchain can adapt to emergent network problems and the changing demands of network users. In an important sense, blockchain users are the ultimate sovereigns because the extent to which individuals choose to store value and transact on a given blockchain determines the valuation of the cryptocurrency. This, in turn, defines the incentives for network participants to verify and process transactions. In this sense, network participants are constrained by the changes to the network that their choices create; if the changes diverge

\footnotetext{
102 Nonetheless, not all cryptocurrency users are happy with the level of centralization that the traditional political institution of delegated representation implies. As compared to a PoW blockchain, EOS is unquestionably centralized, which gives it a greater ability to punish bad actors, as an EOS administrator's correction of a seemingly bad faith transaction indicates. See generally Stephen O’Neal, EOS Proves Yet Again That Decentralization Is Not Its Priority, COIN TELEGRAPH (Nov. 15, 2018), https:/cointelegraph.com/news/eos-proves-yet-again-that-decentralization-is-notits-priority.

103 See Rajagopalan, supra note 3; see also Berg, Berg, \& Novak, supra note 72, at 8-9.
} 
sufficiently from network users' intended purposes, these users can simply use the network less or depart from it entirely.

Cryptocurrency blockchains provide a unique example of private governance whose core characteristics overlap with a number of those typically associated with public governance. ${ }^{104}$ Unlike the traditional centralized hierarchy of firm governance, all participants in the collective output of a given cryptocurrency blockchain can influence the fundamental rules governing this output. Nonetheless, these rules are subject to market choice by users in a way that public governance at the nation-state level typically is not: cryptocurrency users can readily vote with their feet. This means the blockchains supporting cryptocurrencies are a unique case study in private governance, ${ }^{105}$ in which unusually decentralized decisions surrounding fundamental rule sets are subject to competitive market pressures facilitated by relatively low exit costs. These blockchains thus mix governance features traditionally associated with public and private organizations, respectively. Allowing for uniform and clearly regulated input from all participants in the process as to the fundamental rule sets governing them, while simultaneously subjecting this governance process to competitive pressures, is a unique innovation in governance that should pique the interest of institutional scholars and practitioners.

This infers that exit costs greatly define the incentives of individuals choosing to make costly bets on network participation and use. In a context where exit costs are relatively low, the stakes of any given group decision are necessarily lower ${ }^{106}$ (provided, of course, that the group decision itself does not directly implicate exit costs). For anyone who lives sufficiently close to the border of a state rewriting its constitution, the downside risk associated with constitutional change is lower. If the outcomes are sufficiently undesirable for any given individual who faces low moving costs, that individual will likely choose to move rather than incur the costs associated with the change in governance. In practice, though, even moving a few miles across state lines poses a significant cost, especially as compared to the cost of choosing to mine or transact in another cryptocurrency. This simple fact of relatively low exit costs for cryptocurrency users and participants has important implications for the dynamics likely to result from constitutional level governance changes to a given blockchain. Interestingly, though, exit costs are only one side of the ways in which networks can condition membership - entry costs are another option, albeit one that has yet to be encoded

\footnotetext{
${ }^{104}$ See Cowen, supra note 61.

${ }^{105} \mathrm{Id}$.

${ }^{106}$ See Hirschman, supra note 21, at 82-6.
} 
into a cryptocurrency blockchain. ${ }^{107}$ As I have noted, though, Ethereum proposed change to its consensus mechanism directly creates an entry cost for anyone seeking to become a network validator.

One cause of these low exit costs is the nature of blockchain rule changes themselves such as the possibility for a fork that reflects both opposing participants' governance preferences on a given issue also lowers the stakes associated with a given rule change. Whether or not a given set of cryptocurrency participants' governance preference on an issue carries the day, if a fork in the blockchain reflecting their preferences will persist after the rule change, this reduces the costs of such change in governance to the "losers." A second characteristic of cryptocurrency communities also reduces the cost of governance changes to losers; literal exit from participation or use of the cryptocurrency itself. Moving one's stake in a given cryptocurrency blockchain to another permissionless-blockchainsupported cryptocurrency, or into more traditional financial instruments altogether, facilitates exit to a level never enjoyed by individuals who are subject to traditional public constitutional governance.

Because exit costs directly influence the incentives of network participants, these exit costs themselves create a potential margin for competition over governance. Exit costs influence participants' and users' incentives both during periods of ordinary network operation, as well as times when governance changes are being wrought. Similar to a variety of investment contexts, the speed at which one can convert a costly investment into a more liquid asset directly affects individuals' willingness to invest, and, accordingly, the return offered on the investment. ${ }^{108}$ But the effects of exit costs are not limited to the individual incentives of network users and participants. Because exit costs influence the changes in governance that are likely to be realized, network participants have an additional channel by which their incentives are implicated. Is a reduction in network participants and the emergence of a competing but closely related cryptocurrency worth the benefit of the more representative governance that is likely to result in contexts where exit costs are lower? Participants must thus examine how a rule change will affect both their own incentives and the exit

107 Although no major cryptocurrency currently conditions network participation on bonding or a set of personal characteristics (more commonly associated with citizenship), some DAOs have discussed requiring a bond be posted for participation. Relatedly, the feature that led to the DAO hack could be seen as a temporal exit cost, preventing funds from being withdrawn for a set number of days for strategic reasons. See generally Klint Finley, A $\$ 50$ Million Hack Just Showed That the DAO Was All Too Human, WIRED (June 18, 2016), https://www.wired.com/2016/06/50-millionhack-just-showed-dao-human/.

${ }^{108}$ See generally Douglas W. Diamond, Liquidity, Banks, and Markets, 105(5) J. PoL. ECON. 92856 (1997). 
decisions of other participants. They must also consider how comparatively better or worse outcomes will then affect a similar calculus on the part of network users.

Lower exit costs thus facilitate greater competition in governance, which is often argued to result in benefits associated with both enhanced government accountability to citizens and experimental discovery. ${ }^{109}$ Lower exit costs also suggest greater variance in policy change to the extent that constituents self-select into the governance regime that most closely represents their preferences, as opposed to using their input to moderate governance change in a regime in which they are a minority. ${ }^{110}$ However, cryptocurrencies dependent on proof-of-work algorithms need a sufficient number of miners to prevent attacks. These attacks result when one set of network nodes controls more than fifty-one percent (51\%) of the computing power on the network. This means sufficient exit of miners carries costs to the network writ large in ways that are similarly costly to users choosing to stop transacting in the currency altogether. Because of this trade-off in terms of the representativeness of governance, the sufficiency of network participation, and the use to achieve its intended purposes, it is clear that lower exit costs are not uniformly better.

In the context of Ethereum in particular, two things indicate attempts to influence exit costs on the margins in ways that mean participants cannot as easily choose to exit, whether to another Ethereum-based cryptocurrency or to another cryptocurrency altogether: (i) the use of hard forks that do not result in two viable chains $^{111}$ and (ii) the intent to make certain types of computer processors less profitable for network participants. ${ }^{112}$ Another important aspect of exit costs surrounds the relationship between blockchain rule sets and the public legal systems in which blockchain participants and users reside. ${ }^{113}$ EOS has a written constitution that specifies, among other things, forums for dispute resolution among network participants and users. This suggests that the choice of the public legal system as a backdrop for on-chain disputes will prove an additional margin of competition. Importantly, choosing to resolve a blockchain-related dispute before the courts is another exit cost that different blockchain governance regimes will likely compete on.

${ }^{109}$ Id. at 932-943; see also Hirschman, supra note 21, at 3.

${ }^{110}$ See Scott Gehlbach, A Formal Model of Exit and Voice, 18 Rationality And Society 395, 5-6 (Nov. 1, 2006).

111 See O'Leary, supra note 70.

${ }^{112} \mathrm{Id}$.

${ }^{113}$ See Berg et. al., supra note 3, at 5. 
Currently, though, the scope of the rules governing network processes on a given permissionless blockchain is largely up for grabs by the same consensus mechanism. This means that larger rule changes proceed with the same level of consensus from network participants as more ordinary ones. Given the lessons from constitutional design surrounding the necessarily expressive nature of defining and changing a constitution, network participants are likely to increasingly consider rule entrenchment as a design choice surrounding certain governance parameters. The extreme of unamendability is baked into certain network design parameters from the get-go, but otherwise making certain aspects of governance likewise permanent is also an option that can provide a competitive governance margin for blockchain network designers, as well as network participants in terms of updating pre-existing governance choices.

In sum, there are a number of competing forces resulting from governance choices that will greatly influence the number of viable cryptocurrencies: adoption, competition, and exit costs for participants and users. The need for sufficient adoption acts as a downward pressure on the number of cryptocurrencies; past a certain point, too many cryptocurrencies with tiny user bases will prevent their intended uses from being successful or will outright vitiate the benefits of permissionless blockchains altogether by making a fifty-one percent $(51 \%)$ attack too easy. Competition between blockchains as a function of their outputs will similarly reward and discipline blockchains whose governance choices on these margins will determine their success and failure. ${ }^{114}$ Finally, the low costs of exit both facilitate competitive pressures and present a direct governance design choice on which cryptocurrency blockchains can compete. Do cryptocurrency users and participants prefer the lower exit costs associated with forking the blockchain and moving to other currencies, or can barriers to exit actually improve on-chain governance outcomes?

These trade-offs can be understood by considering one example based on the currently dominant cryptocurrencies' governance structures. Rigidities in these governance structures lend most of the benefits in terms of their use as currencies but have revealed a hidden set of costs surrounding the inability of these currencies to adapt to changing demands (to which some users would argue they should not adapt). This implies a competitive benefit to more blockchains whose governance models are slight to significantly different. In the long run, this should lead to more efficient blockchain governance, but it implies a trade-off for the design of blockchains themselves, as well as the market more broadly. Is a greater diversity of blockchains more efficient, or are fewer, more flexible blockchains ideal? While

${ }^{114}$ Cowen, supra note 61. 
flexibility has been linked to constitutional endurance, it is not as clear that the mutability of network rules is the right competitive margin for blockchain participants and users, especially as compared to exit costs. Blockchains' value is only realized after a sufficient number of users adopt the use of cryptocurrency that a given blockchain supports. This suggests diminishing marginal returns to numerical competition in governance; the benefits of competition in governance between different blockchains cut against the benefits of scale that clearly exist in blockchains more generally. This means the optimal level of flexibility in blockchain governance can be seen as a function of the number of permissionless blockchains out there in a particular industry. Past a certain point, this could cut toward the need for fewer blockchains with more flexible governance mechanisms, including exit costs calibrated to make one's participation and use of a cryptocurrency more or less "liquid."

A related trade-off surrounds the current nature of governance change that can fork the blockchain into two chains of code, which could be construed as facilitating competitive governance processes, or as stymying them. Firm governance is subject to competitive pressures. In theory, blockchain forks create a possibility analogous to that of cloning a firm and subjecting it to two distinct governance structures. However, in the case of a blockchain forking, it is not as if a randomized controlled trial in governance is occurring with respect to a single firm. Once a cryptocurrency has forked, the two new currencies are by and large competitors. ${ }^{115}$ Furthermore, competition among cryptocurrencies is occurring on a single originating blockchain, which creates an additional margin for competition. Is a cryptocurrency's ability to compete with other cryptocurrencies diminished when it forks? Put differently, is its brand diluted when this occurs? ${ }^{116}$ A further interesting possibility surrounds the ability of blockchain participants and users to hold or divest the different currencies sometimes resulting from a hard fork. Someone holding Bitcoin and Bitcoin Cash could exchange all of their holdings in one currency for holdings in the other as soon as these transactions could be processed and validated after the fork occurred. This means prices of the distinct cryptocurrencies subsequent to the fork can (at least in theory) reflect participants' and users' expected valuations of the underlying rule changes. This is only true to the extent that blockchain participants and users are sufficiently and accurately informed as to the underlying governance trade-offs described here, though.

\footnotetext{
${ }^{115}$ In theory, a cryptocurrency blockchain fork could occur to create complementary currencies, with the underlying distinctions in blockchain governance deliberately designed to create networks whose capabilities at a minimum do not compete with one another and at most would be direct complements to one another. Thus far, though, existing forks in cryptocurrency blockchains have not occurred with the express intent to achieve these outcomes.

${ }^{116}$ See Arvind Narayanan, supra note 49, at 172-173
} 
Thus, it should be noted that the arguments contained herein about network participants' and users' input to governance depend upon awareness on the part of these individuals of potential changes in governance and the costs and benefits thereof. Accordingly, when considering blockchain governance changes, it is worth asking how many blockchain users are sufficiently familiar with these debates to be able to exercise a costly informed choice along the margins of governance variation described thus far. In the case of network participants, incentives are wellaligned for them to be informed as to these trade-offs; participants' input is required at a high level for changes to occur, and the continued value of participants' costly investments in electricity and hardware depend significantly on the rules governing the creation of additional cryptocurrency. ${ }^{117}$ In the case of users - those who simply invest and transact in a given cryptocurrency - it is unclear just how well informed they might be about the costs and benefits of proposed governance changes. Nonetheless, because of their direct effect on the liquidity and value of cryptocurrency investments, exit costs are arguably among those most likely to be salient even to ordinary users of cryptocurrencies. If these costs increasingly become a competitive margin, this suggests there may be increased variance in governance in cryptocurrency blockchains, as one chain increases exit costs to retain its existing user and participant base, and another keeps exit costs low at the cost of losing a subset of its users and participants, at least in the short term.

Granted, notwithstanding some level of ignorance on the part of users and participants as to the governance changes being debated, changes in the market valuation of cryptocurrency often follow the success or failure of a given proposed governance change. Nonetheless, given that network participants are by definition users - at least for the period following their successful mining of a given amount of the underlying cryptocurrency-their choices following successful and unsuccessful governance changes are likely to inform price signals accordingly. However, network participants account for only a fraction of network users. Furthermore, cryptocurrency price shocks occur for a variety of reasons other than governance changes, such as regulatory changes in countries with high levels of crypto users, or frauds or hacks of major exchanges. This paper is therefore intended to clearly spell out the economic and institutional trade-offs implicit in the seemingly esoteric governance debates occurring among cryptocurrency network participants.

${ }^{117}$ See Berg et. al., supra note 3, at 5 


\section{CONCLUSION}

These debates over the best set of rules about making rules, or more typically, constitutions, are not an understudied topic. But in the context of private governance-where rule sets are typically more fluid, centrally controlled, and exist in the shadow of law and regulation - developing generalizable insights about comparatively superior governance mechanisms is more difficult. Permissionless cryptocurrency blockchains provide a fruitful context within which to explore these questions. These blockchain ecosystems can be understood as a type of constitutional rule set that both defines and legitimizes the activities supported by permissionless distributed ledger technology. The development of cryptocurrency blockchains has thus led to new forms of competition in private governance, which include exit costs as one of the fundamental margins upon which governance outcomes will be shaped between blockchains. Although less salient in design proposals currently, entry costs and rule entrenchment are also likely to play an increasing role as different chains continue to tweak their governance choices in light of the demands of network participants and users.

Constitutional and political theorists should therefore neither be baffled by nor disinterested in the innovation currently occurring in governance processes on permissionless blockchains. These processes share many similarities to constitutional processes, and insights from constitutional design surrounding implementation and amendment shed considerable light on current developments in the communities supporting major cryptocurrency blockchains. The trade-off between flexibility and rigidity has caused divides in these communities as they debate how best to scale network processes while remaining true to the collectively expressed understanding of the constitutional rule set of each blockchain. Some changes to network processes that require the assent of a sufficient proportion of network participants do not fundamentally alter the rules by which network processes are governed. Instead, they affect the costs-and-benefits network participants expect as a reward for facilitating network processes of currency creation, validation of transactions, and participation in ongoing governance. Changes to data block size, hash function difficulty, transaction fees, and reward for hash function resolution all stand as examples of this type of constitutional change on the blockchain. Other changes implicate more fundamental additions or alterations to governance processes, such as the definition of subsidiary governance or an overhaul of the entire process of governance change itself. While the Bitcoin network's most salient proposed changes have limited themselves to, at most, creating a sublayer of governance, Ethereum's proposed changes not only include a related form of decentralized governance but also involve wholesale changes to the means by which future changes to network processes will occur. 
The study of public and private governance is now ancient. Thus, while blockchain processes present unique expressions of governance questions in new contexts, it is not as if challenges in obtaining consensus surrounding a given group's processes and governance are new. This means that an understanding of the fundamental trade-offs associated with constitutional design questions, with a particular focus on amendment processes, could considerably benefit blockchain designers and participants. Flexibility in amendment is seen as allowing necessary adjustment absent which an entire constitutional order can be overturned. Nonetheless, amendment thresholds should be sufficiently high that fundamental rules are insulated from ordinary pressures. Constitutions are seen as trading off between facilitating economies of scale in governance while simultaneously creating agency problems among those individuals who provide direct input to legislative, executive, and judicial governance. Decentralization is one solution that allows for better representativeness while preventing a level of centralization that obviates the ability to govern the number of processes demanded by a given nation's constituents. Thus, the relationship between blockchain participants and users as a function of the processes governing them is clarified through understanding the trade-offs that have long been debated in the context of constitutional design and subsequent amendments.

Finally, decentralized processes similar to those of governance via executive, legislative, and adjudicative functions may never have been automated at the economic scale and complexity seen on permissionless cryptocurrency blockchains. Blockchains can, therefore, be seen as an early example of constitutional law as code. ${ }^{118}$ This means that competition among blockchains, which results in unique governance solutions, could be a valuable and informative precursor to the application of automated governance in the public sector. The terms of blockchain network participation and use that define entry and exit will become important margins of competition that shed light on the myriad institutional forms of digital citizenship and exit that are likely to emerge across the 21 st century. Of course, it should be emphasized in conclusion that the benefits of this competition are only attainable if there is sufficient informed adoption of cryptocurrencies to where the competitive pressures described here yield efficiency-enhancing governance benefits.

${ }^{118}$ See Primavera de Filippi \& Aaron Wright, supra note 35, at 194. 